\title{
ORMS DOPPELZEICHEN BEI SWEET UND BEI MORSBACH.
}

In zwei kleinen aufsätzen (Anglia VII Anz. 94 ff. u. 208 ff.) glaub ich erwiesen zu baben, dass Orm mit seinen doppelstaben nicht, wie bis dahin allgemein angenommen worden war, kurze des vorhergehenden vocals andeuten wolle, sondern consosantenlänge ausdrtickt.

Gegen meine darlegungen richten sich, wenn anch mein name nicht genannt wird, einige zeilen in Sweets First Middle English Primer (1884) und die absätze 616 und 617 in dessen History of English Sounds (1888). Die zweite und ansfthrlichere stelle setz ich vollständig hierher, zerlege sie aber zur bequeneren besprechung in ibre funf hauptbestandteile; sie lautet

$\therefore$ In this [Orm's] text every consonant that is final or followed by another consonant is doubled after a short vowel, as in patt, crisstenndōm, inn $=\mathrm{OE} i n n$, in. This shows that the $\mathrm{OE}$ distinction between in and inn must have been lost in pronunciation as well as in writing, so that all final consonants were lengthened after a short vowel, as in Modern English. Such spellings as ic amm, scipp = eom, scip oceur already in Du. [= Durham Gospels], and many examples might be quoted from later texts. Ld [= Laud Chronicle] has namm preterite, iett 'yet' etc., godd occurs in Layamon, Juliana, Genesis and Exodus, Cursor Mundi.

: The opposite tendency is to shorten long consonants after a long vowel; and so double final consonants came to be associated with preceding short vowels. 
and single consonants with long ones in writing as well as in speech. There are isolated traces in $\mathrm{OE}$ of Orms doubling of a consonant before another consonant in such spellings as follce, illce (Smith's Bede), where, however, the doubling may really indicate cons. length. In effter (Juliana, Ayenbite) the doubling of the $f$ may mean voicelessness.

3. That Orm's doubling did not mean cons. length, but had been reduced to an abstract symbolization of vowel-length, as in Modern English and German, is clear from his extension of it to unstrest syllables, as in brōperr, for already in $\mathrm{OE}$ double conss. are shortened after an unstrest vowel, whether long or short, additional ME examples being afforded by such spellings as leofmones 'lemman's' = OE lēofmannes, meinfule $=\mathrm{OE}$ mogen-fulla in Juliana, sunfule $=$ synnfulle in Ancren $\mathrm{R}$.

4. Where the consonant is followed by a vowel, as in sune $=\mathrm{OE}$ sunu, it was not possible to double the consonant, because it would have been pronounced double, and sune would have been confounded with sunne 'sun'. That sunne was really pronounced with long or double $n$ is proved by the metre, which allows sunne to come at the end of the verse, and rigorously excludes sune from that position.

5. Here then Orm's clumsy spelling breaks down completely, and he feels this himself, for he often marks the shortness of the vowel in such words as sune with a ( ), as in takkenn, name chèle = OIcel. taka, OE nama, cęle. Often, too, he marks length with the old accent, as in láre $=\mathrm{OE}$ lár, which he of ten doubles, or even trebles, especially before $t$ as in $u t$.

Zu 1. hab ich zu bemerken: Die längung auslautender consonanten in worten wie god, lof, scip trat schwerlich erst im Spät-altenglischen ein, sondern muss schon ziemlich frth geläufig gewesen sein. Dies ergibt sich aus dem versbau; denn verse wie Beo. 478 gód éaðé mág und Phoen. 394 wórhte wér ond wî́f sind nur richtig unter der bedingung, dass das $d$ in god und das $r$ in wer lang war. Dass das hauptwort 
inn oft, das vorwort in nie mit zwei $n$ geschrieben wird, erklärt sich daher, dass das erste ein sinnstarkes, das zweite ein sinnschwaches wort ist. Wie noch im Neuenglischen verbinden sich im Altenglischen sinnstärke und nachdruck mit langem consonantischem auslaut und sinnschwäche und fluchtige aussprache mit kurzem consonantischem auslaut (vgl. die bemerkungen zu 3). Das dingwort inn war als solches immer sinnstark und hatte deshalb immer langen consonanten; das vorwort in war als solches meist sinnschwach und hatte, so oft es dies war, kurzen consonanten. Erhielt es einmal gewicht, wie in dem verse Scéde-lánduim in, so ward es auch mit langem consonanten gesprochen; die schreibung aber blieb um so mehr bei einfachem $n$ als sogar worte wie $\operatorname{man}(n)$, næs $(s)$, bed $(d)$ meist mit einfachem schlussconsonanten geschrieben wurden.

$\mathrm{Zu}$ 2. bemerk ich: Es ist ganz richtig, dass die neuenglische und neuhochdeutsche weise, kturze des vocals durch zweifachen consonanten anzudeuten, ihren ursprung darin hat, dass vor langen consonanten meist kurze vocale standen und lange vocale vor langen consonanten kurz zu werden pflegten. Aber bei Orm und im gleichzeitigen Englisch ist die spätere tubung, kurze des vorhergehenden vocals durch doppelconsonanten anzudeuten, so wenig eine bewusste und mit absicht angewante regel wie in irgend einer andren germanischen sprache um das jahr 1200.

Zu 3. ist zu sagen: Niemand hat bestritten und kann bestreiten, dass schon in der ae. zeit lange consonanten in untreffigen silben kurz werden (wēstenes aus wēstennes, ópere aus operre); es ist auch ganz richtig, dass später auch nebentreffige silben von der kurzung ergriffen werden (lēofmones statt lèofmonnes, sunfule statt sunfulle). Falsch aber ist, wenn Sweet behauptet, Orms consonantendoppelung erweise sich dadurch, dass er sie auch auf untreffige silben anwende, als blosses mittel die kurze des vorhergehenden vocals auszudrticken.

Sweet weiss so gat wie ich, dass z. b. in dem verse Miltons exhausted, spiritless, afflicted, fallen die silben -ted, -less, -len (wenn -len als besondre silbe gesprochen wird) im feierlichen vortrage auf langen consonanten ausgehn. Sweet weiss auch, dass in gewöhnlicher rede untreffige silben, sobald sie einiges gewicht erhalten, mit langen consonanten gesprochen Anglia. N. F. VI. 
werden. Did he begin? No, Sir, he ended! In diesem berichtigenden und mit nachdruck gesprochnen ended ist das erste d kurz, das zweite, obwol es in untreffiger silbe steht, lang: die untreffige silbe -ded nimmt teil an dem gewichte des wortes ended, wodurch sie selber etwas nachdruck bekommt, und unter diesem nachdrucke längt sich ihr auslautendes $d$. Doch Sweet ist ein so grundlicher kenner dieser dinge, dass es keinen sinn hat, sie ihm auseinander zu setzen; ich will deshalb nur feststellen: dem Neuenglischen sind lange consonanten auch in untreffiger silbe nicht ungeläufig; und sie treten immer ein, sobald eine untreffige silbe aus irgend einem grunde gewicht erlangt.

Und wie im Neuenglischen so im Altenglischen. Zwar die alltägliche ae. rede, die wir nur unvollkommen und aus der schrift kennen, lehrt uns hier nichts; wol aber tat es die gebundene rede, in so ferne wir ihren rhythmus kennen. Z. b. in den versen éllén frémedón, Scéde-lúndưm in, cásér-dómés, hringéd býrné haben die silben -len, -don, -dum, -ser, -mes, -ged je einen ganzen takt zu fullen, sind folglich lang. Durch den vocal können sie es nicht sein; es bleibt also nichts tubrig als zu schliessen, dass sie es durch den auslautenden consonanten sind, oder dass -len, -don, -ser usf. -lenn, -donn, -serr usf. lauteten. Also auch das Altenglische sprach lange consonanten auch in untreffigen silben, sobald diese gewicht erhielten. Steht nun fur das Ae. wie fur das Nenenglische der gebrauch langer consonanten auch in untreffigen silben fest, so gibt es 'keine möglichkeit, dem Mittelenglischeu die selbe eigentumlichkeit abzusprechen. Und waren dem Mittelenglischen lange consonanten auch in untreffiger silbe gelänfig, so sind wir genötigt anzunehmen, dass Orm mit seinen doppelzeichen, durch die er in treffigen silben stets consonantenlänge ausdrtickt, auch in untreffigen consonantenlänge meine.

Nun bat sicher die doppelung der auslautenden consonanten nicht bloss gewisser sondern äller untreffigen silben auf den ersten blick etwas befremdliches; doch nur auf den ersten blick, und sie ist keinesweges unerklärlich: Orm schreibt in versen and sprach and wollte jede silbe seines eben and wurdevoll einherschreitenden verses mit nachdruck gesprochen wissen.

Und es ist nicht schwer za sehn, wie Orm za dieser her- 
vorhebung auch der untreffigen silben kam. Seine langzeile ist die abkommin der alten s abzeile, wie verschieden sie auch von dieser ist. Die entfernung von der alten weise ist gewirkt worden durch den einfluss der lateinischèn kirchenhymne. Der vers dieser hymne, in der zahl der takte dem germanischen verse gleich, unterschied sich wesenlich im bau der takte: im lateinischen verse jeder takt zweisilbig, d. h. hebung and senkung je durch eine silbe vertreten; im germanischen verse dagegen der takt nach belieben ein- oder zweisilbig, d. b. hebung und senkung bald durch eine bald durch zwei silben vertreten. Der lateinische vers hatte dadurch ebenen, der germanische holpernden (tumbling, sagt könig Jakob) gang. Durch das jahrhunderte lange eindringen des hymnenverses anf die germanischen ohren gewöhnten sich diese an den ebenen rhythmus, und die germanischen dichter fingen an die einsilbigen takte zu bekämpfen. Der vers unsres Otfrid, der um die mitte des 9. jahrhunderts schrieb, ist der erste erfolgreiche schritt anf diesem wege. Bei den Engländern tauchen ähnliche bestrebungen nicht viel später auf. Im Poema Morale, das vor dem jahre 1200 entstand, ist der ebene rhythmus des lateinischen verses beinahe erreicht; Orm vollendet die bewegung ohne jedoch, wie der schluss seiner langzeile zeigt, ganz von der germanischen weise los zu kommen (sieh Anglia V Anz. s. 124-125). So weit geht Orm in der anbildnng des heimischen verses an den lateinischen, dass er lieber den worttreff verletzt als einsilbigen takt bildet, und dass ihm tref figungen, wie sie die verse afftérp pe fléshess kindé, pa góddspelléss neh állé, to bén undérr deofléss peowwdbm enthalten, so wenig anstössig sind wie entsprechende des lateinischen verses. Wenn er nun schwache silben, die durch den rhythmus za starken werden, wie starke schreibt, so verfährt er nur folgerichtig. Noch eine eigenttumlichkeit des hymnenverses bildet Orm nach. Der durch Gregor eingefuhrte gesangstil hob die bis dahin herrschend gewesene metrische dichtung auf: die neue weise schied nicht mehr zwischen langen und kurzen, sondern nur noch zwischen treffigen und untreffigen silben; und hebung und senkung hatten in den neuen versen gleiche daner, so dass z. b. vení creátor spiritús nicht war

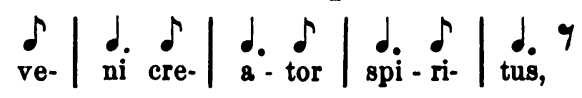


sondern

$$
\left.\begin{array}{c|cc|cc|cc|cc}
d & d & d & d & d & d & d & d & d
\end{array}\right]
$$

Dem frommen Augustiner Orm waren natturlich nur dieser gesang und diese verse gesang und verse. Um seinen versen den selben sanften und gleichmässigen fluss zu sichern, musste er futr gehörige hervorhebung auch der untreffigen silben sorgen. Die sprache begunstigte die lesung nu bróbęr Wáltęr brópęr min - die punkte bezeichnen fluchtige aussprache der betr. silben -, während das lateinische vorbild nù brópèr Wáltèr brópèr min forderte. Verstärkte sich aber das gewicht der untreffigen silben, d. h. wurden sie treffigen ähnlich und sogar gleich, so mussten sie den ausspracheregeln der treffigen unterliegen und handelte 0 rm wieder bloss folgerichtig, wenn er nu broperr Wallterr broperr min schrieb. Dies ist die naturliche und einfache erklärung der doppelstaben Orms in nebensilben.

An dem was Sweet unter 4. behauptet ist richtig, dass sunne von Orm mit langem $n$ gesprochen ward und sune, weil es kurzes $n$ hatte, nicht den ausgang der langzeile bilden konnte. Ganz verwerflich aber ist die meinung, dass Orm in sune nur deshalb die kurze des vorhergehenden vocals nicht durch doppelung des $n$ anzeigte, weil es sonst mit sunne zusammengefallen wäre. Orm wollte durch seine doppelstaben gar nicht die kurze und durch einfache die länge des vorhergehenden vocals ausdrticken! Er wäre ein dummer kerl gewesen, wenn er es gewollt hätte; denn er hätte zu mitteln gegriffen, die schon einem andren zwecke dienten: er hätte doppelzeichen gesetzt bald um kturze des vorhergehenden vocals anzudenten (wissdom; broperr, floeshess), bald um langen consonanten auszudrtlcken (mann, bedd; godd, bacc; sunne, fille); und er hätte in boc win hoepen usf. einfache staben gesetzt weil, und in sune micel hider usf. ebenfalls einfache, obgleich der vorhergehende vocal kurz war. Nein, alter Orm, diesen vorwurf lass ich nicht auf dir sitzen! Warst du auch kein grosser dichter, oder vielmehr uberhaupt kein dichter, so hattest du doch feinen sprachsinn und einen klaren kopf: deine doppel-

1 Sieh z. b. R. von Liliencron in Pauls Grundriss der Germ. Phil. IIb, $310-311$. 
staben bezeichnen jedes und alle mal langen und deine einfachen jedes und alle mal kurzen laut! Und da deine schreibung, so aufgefasst, nicht nur verntunftig in sich und durchaus folgerichtig ist, sondern auch im besten einklange mit der geschichte der sprache und mit der metrik steht, so ist sie eben so) und nicht anders aufzufassen!

Von dem was Sweet unter 5. sagt, ist jedes wort zu verwerfen. Orms schreibung ist keinesweges ungeschickt, klobig, ungeschlacht, oder wie man Sweets clumsy thersetzen will, sondern, wie eben gesagt, verständig, zweckmässig und folgerichtig. Und sie bricht durchaus nicht in sich zusammen, indem sie in worten wie sunne, die kurzen vocal haben, doppelzeichen setzt, and in worten wie sune, die ebenfalls kurzen vocal haben, einfache zeichen, sondern Sweets auslegung der schreibung Orms geht in scherben: Orm bezeichnet mit seinen doppelstaben eben nicht bald lange consonanten bald kturze des vorhergehenden vocals, sondern stets und therall lange consonanten. Orm hat auch ganz und gar nicht das gefthl, dass seine schreibung der consonanten nichts tauge, und dass er um deswillen mit den bekannten häkchen zur bezeichnung der kurze und strichen zur bezeichnung der länge nachhelfen musse, sondern hat für die anwendung dieser abzeichen andre und leicht ersichtliche gründe. Der erste grund ist, gleich geschriebene und nur durch die daner des stammvocals verschiedene worte auch dem ange sofort als verschieden kenntlich zu machen; daher tākenn 'zeichen' neben tăkenn 'nehmen', nāme 'du nahmst' neben năme 'name', lāte 'aussehn' neben lăte 'späte', hēre 'hier' neben hĕre 'heer' und 'ihre', cōme 'ankunft' neben cŏme 'komme', rèle 'fuhle' neben fèle 'viel', dāle 'teile' neben dăle 'tale'. In andren als solchen worten kommt das zeichen fur die kurze kaum vor. Das längezeichen gebraucht Orm, ausser zu dieser unterscheidung noch zu einem andren, doch ebenfalls leicht erkennbaren zwecke: er setzt es ein-, zwei-, ja dreifach tiber den vocal einer anzahl anf $t$ ausgehender worte: $\bar{u} t$ 'aus',

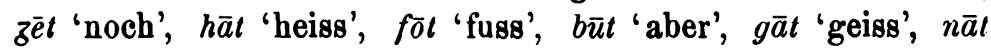
'weiss nicht'. Alle diese worte haben in ne. mundarten, z. t. auch in der ne. gemeinsprache, den ursprtinglich langen stammvocal kurz. Dass Orm einen, zwei und sogar drei längestriche uber diese vocale setzt, erklärt sich sehr einfach wenn man 
annimmt, dass die neigung zu kturzen schon im anfange des 13. jahrhunderts eingesetzt hatte, und dass Orm mit seinen strichen sagen will: "Das wort fot (gat, ut, zet usf.) hat langen vocal; zwar manche leute, die nicht ordentlich Englisch können, sagen föt, aber du, freundlicher leser, wirst fōt sprechen!"

Als kämpfer fur Sweets unhaltbare lehre erhebt sich jetzt L. Morsbach in $\S 15$ seiner Mittelengl. Grammatik, derer erste hälfte eben erschienen ist. Morsbach steht ganz auf Sweets boden. Wie Sweet gibt er za, dass Orms doppelstaben auslantend and in worten wie sunne, sette, wisse lange consonanten darstellen. Wie Sweet hält er die länge des endconsonanten in godd, shall, loff usf. für erst in spät-altenglischer zeit entstanden. Mit Sweet glaubt or, dass Orm die kturze des vorhergehenden vocals in sune, micel, godes usf. nur deshalb nicht durch doppelzeichen andeute, weil dies zu falscher aussprache verfuhrt hätte. Sweets haupteinwand, Orms verwendung von zweifachen staben auch in untreffigen silben beweise unwiderleglich, dass er vocalkürze damit andeuten wolle, ist auch Morsbachs haupteinwand. Wie Sweet glaubt Morsbach, Orm habe zuweilen die ungewissheit und unzulänglichkeit seiner schreibung empfunden und dann kturzehäkchen oder längestriche zu hilfe gerufen. Morsbach macht aber auch zwei eigne einwände.

Der erste ist: "Dass Orm nicht die länge oder kturze der consonanten, sondern der vocale bezeichnen wollte, geht zur gewissheit aus seiner schreibung der diphthonge hervor: aw für $\bar{a} u$ neben awn für au, cen für $\bar{e} u$, en eon für $\bar{e} u$ neben ewn eown fur eu, on fur ōu neben own für ou, ferner azz fur $a i$, ez子 ftur ei z. b. in sārvle clawwess, shöenwenn, nēwe, cnewwe, flōwenn trowwenn, dazz, wezze."

Aber wenn $z \zeta$ und ww wirklich nur die ktirze des vorhergehenden vocals bezeichnen, wie kommen dann worte wie wezzess und trowwenn 250 mal, oder öfter, in den versausgang, den doch nur worte der form $\dot{-} \times$ bilden können? Es gibt nur eine antwort: wezze, twizzess, trowwenn, cnewwess usf. sind, eben weil sie im versausgange stehn, so gut wio sunne, lasste, kêpenn usf. worte der form $-\times$; da aber ihre stammvocale kurz sind, muss in den $3 \zeta$ und $w w$ das sticken was sie befahigt den versansgang zu bilden, $d$. h. mtissen diese doppelzeichen lautlänge darstellen gerade wie es $m m, t t, s s$, 
If usf. tun, und können sie so wenig wie diese bloss den zweck haben, die kurze des vorhergehenden vocals anzudeuten. Und so ist es; nur darin unterscheiden sich $3 z$ und ww von den ubrigen doppelstaben, dass sie nicht lange consonanten bezeichnen, sondern lange vocale: $z \zeta$ bedeutet $\bar{\imath}$, und $w n$ bedentet $\bar{u}$.

Woher die langen $i$ und $u$ kommen, ist bei geschlossnen silben ohne weiteres klar. Ae. doeg und cneon sind, wie wir gesehn haben, so viel wie dagg und cneoww', d. h. enden anf lange consonanten. Werden lange $g$ (lantlich $=y$ in yes) und lange $w$ (lautlich $=w$ in way) zu vocalen, so können sie nattirlich nur lange $i$ und $u$ geben. Doch auch die länge der $i$ und $u$, die aus anlautenden $y$ und $w$ (doe-ges, cneo-wes) entstanden sind, ist nicht unerklärlich; and wir werden uns ihre entstehung so zu denken haben: Die alten $g$ und $w$ wurden nicht mit einem schlage zu $i$ and $u$, sondern gingen durch die zwischenstufe iy and uw. Der vocalische bestandteil schlug sich zur ersten, der consonantische zur folgenden silbe. Allmählich schwand der consonantische; doch noch ehe dies geschehen war, hatte sich der vocalische, und zwar weil er silbenauslant nach kurzem vocal war, gelängt ${ }^{2}$. Der weg wäre also gewesen: da-yes dai-yes dā̄-yes daī-es und cla-wes clau-wes clau-nes claū-es. Sweet hat schon 1877 (Handb. of Phon. $\S 188$ und 200) gut und treffend das folgende ther die silbe und den silbenauslant im Neuenglischen bemerkt: "In English final consonants are long after short, short after long vowels.... Even stops [ = klapper, verschlusslante] are lengthened finally after short vowels .... The lengthening of the glide $[=$ des zweiten bestandteils] in eye is to compensate for the shortness of the vowel: if both vowel and glide were short, we should have a short monosyllable, which is contrary to the general character of English". Durften wir annehmen, dass schon das Mittelenglische von demselben gesetze beherrscht ward, welches Sweet hier fur das Neuenglische aufstellt, so wären die langen silbenauslautenden $\bar{\imath}$ und $\bar{u}$ Orms (wezz-es, troww-en) aufs beste erklärt. Und wir dturfen es getrost; denn die langen silben-

1 Ich stehe, indem ich die stammvocale von cneow-, treow-, clawund andren kurz ansetze, zu Kluge, Cosijn und Luick, stiltze mich aber ausser auf die von ihnen angefuhrten grtinde auch auf metrische tatsachen. Orms trowwen führ ich auf ae. trŭwian zuritck.

2 Aehnlich denkt sich die sache Luick, Anglia Beibl. IV, s. 107. 
auslautenden $i$ und $u$ nach kurzen vocalen stimmen trefflich zum allgemeinen charakter des Mittelenglischen, zunächst der sprache Orms, die, ganz wie das Neuenglische, langen consonantischen auslant nach kurzem und kurzen consonantischen auslaut nach langem vocal setzt.

Also auch $z \zeta$ und $w w$ sollen keinesweges vokalkturze andeuten, sondern drticken langen vocal aus; und nach kurzen vocalen finden wir $z \zeta$ and $w w$, nach langen vocalen $z$ and $w$ durchaus nicht, weil Orm die dauer des vorhergehenden vocals anzeigen wollte, sondern weil sich nach kurzen vocalen langer laut gebildet hatte und nach langen vocalen die alte kurze geblieben war. Auch hier erweist sich Orms schreibung von einfachen und doppelzeichen als den tatsachen entsprechend und folgerichtig.

Dies ist sie auch, wenn Orm die zwielaute ei und au der dem Nordischen entlehnten worte (pezzm, hezzlen, nownt, dowwnen usf.) durch ezz and oww darstellt, und wenn er lateinisch-griechische au und eu (Awwstin, Ewwticus) mit awn und eww schreibt: er sprach diese fremden zwielaute mit englischer zunge und schrieb wie er sprach.

Den tatsachen wird Orm auch gerecht, wenn er die endung -ig nicht -izz schreibt, sondern -ið̄. Hālig war schon vor seiner zeit $h \bar{a} \bar{\imath}(\bar{\imath}$ aus $\grave{\imath}+\grave{\imath})$ geworden, und Orms $-i \bar{z}$ ist $\bar{\imath}$; die schreibung haliz̧ wäre halı̌̃ gewesen und hätte nicht die wirkliche aussprache dargestellt.

Aber wie kommt Orm dazu, da es sich doch um vocale handelt, die zeichen $z$ and $w$ zu verwenden, die sonst, wenigstens im anlaute, consonanten darstellen?

Mehr als ein grund musste ihm diese schreibung nahe legen. Zunächst waren die neuen $i$ und $u$ aus $z$ und $w$ entstanden und wurden sie zu seiner zeit noch ganz gewöhnlich durch diese zeichen dargestellt. Dann mögen die neuen $i$ und $u$ bei Orm noch auf der vorhin angenommenen mittelstufe (iy und uw) gestanden haben. Ausserdem empfahlen sich ihm die consonantischen zeichen, weil sie die durchfurung seines grundsatzes, kurzen silbenauslaut durch einfaches, langen silbenauslaut durch doppelzeichen auszudrttcken, möglich machten. Endlich mussten sie ihm genehm sein, weil der gebrauch der zeichen $i$ und $u$ seinen vers, auf dessen festigkeit und klarheit er so grossen wert legte, unsicher gemacht hätten: Orm hat in jedem 
verse, von den 'stummen' $e$ und der verbindung eo abgesehn, so viele vocale wie silben. Hätte er geschrieben 0 daiess and o nihhtess und swa summ Sannt Austin sette, so hätten, da in seinem verse eine um die andre silbe treffig ist, neune von zehn lesern $d a-i$-éss and $A$-ú-stin nach vorbildern wie 12773 Beppsáydá zeháténn, 14268 inn áll patt bóc patt Móysés, 14902 Saúl wass hófenn úpp to king, 15545 Cafárrnaúm zeháténn gesprochen und damit den vers verhunzt.

Morsbachs zweiter einwand ist: "Znsammengesetzte and abgeleitete wörter schreibt Orm in manchen fällen wie die einfachen; so z. b. wippūtenn, wipbinnenn, efennald, efenninng (neben effninng) usf. Hier sind die consonanten $p b, n n$ entschieden kurz, wie die englische sprachgeschichte und anch die schreibung Orms in anān neben onnān, onn ān zeigt. Wenn Orm mit seinen doppelconsonanten wirklich consonantenlänge bezeichnen wollte, so musste er solche schreibungen, die sich an die entsprechenden simplicia wibp, efenn usf. anlehnen und auch beim syllabieren nicht gelängt werden durften, durchans vermeiden."

Auch mit diesem einwande ist es nichts, und Morsbach hätte besser getan ihn nicht zu erheben. Die paar beispiele die er anfuhrt, und die sich noch anfuhren liessen, beweisen gar nichts fur seine und Sweets ansicht, sondern zeigen bloss, dass der gute Orm zuweilen in seiner schreibung ein bisschen sprachgeschichte treibt, und dass sich selbst ein so sorgsamer mann wie er ab und zu verschreiben kann.

So wenig wie Sweet hat Morsbach auch nur den schatten eines beweises dafur erbracht, dass Orm mit seinen doppelzeichen kurze des vorhergehenden vocals andeuten wolle. Ich bleibe deshalb bei meiner bisherigen ansicht; und dem Anglia VII Anz. s. 98 anfgestellten satze, "Orm schreibt auf grund des gesetzes: consonantischer silbenauslant ist kurz nach langem und lang nach kurzem vocal", diesem satze fug ich den neuen hinzu: "Zwielautschliessende $i$ und $u$ sind bei Orm kurz nach langem und lang nach kurzem rocal; die kurzen zwielautschliessenden $i$ and $u$ schreibt er $z$ und $w$, die langen $z z$ und $w w "$.

Bonn.

Moritz Trautmann. 